Research Article

\title{
Alterations in Mucin-Associated Gene Expression on the Ocular Surface in Active and Stable Stages of Atopic and Vernal Keratoconjunctivitis
}

\author{
Mariko Horinaka, Jun Shoji $(\mathbb{D}$, Akiko Tomioka, Yukiko Tonozuka, Noriko Inada, \\ and Satoru Yamagami
}

Division of Ophthalmology, Department of Visual Sciences, Nihon University School of Medicine, 30-1 Oyaguchi-Kamicho, Itabashi-ku, Tokyo 173-8610, Japan

Correspondence should be addressed to Satoru Yamagami; yamagami.satoru@nihon-u.ac.jp

Received 11 March 2021; Accepted 25 May 2021; Published 31 May 2021

Academic Editor: Kofi Asiedu

Copyright (c) 2021 Mariko Horinaka et al. This is an open access article distributed under the Creative Commons Attribution License, which permits unrestricted use, distribution, and reproduction in any medium, provided the original work is properly cited.

\begin{abstract}
Purpose. To evaluate the presence of ocular surface mucin in patients with atopic and vernal keratoconjunctivitis (AKC/VKC), we investigated the mRNA expression levels of SAM-pointed domain-containing ETS-like factor (SPDEF) and mucin-related genes on the ocular surface. Methods. Nineteen patients with AKC or VKC were divided into two groups based on the severity of the disease as determined by their clinical scores for AKC/VKC: the stable group and the active group. Impression cytology was performed in all patients using filter paper, and the expression levels of SPDEF, MUC1, MUC4, MUC5AC, MUC16, and eotaxin-2 mRNA were determined by real-time reverse-transcription polymerase chain reaction. Results. The results showed that the expression levels of SPDEF and MUC5AC mRNA in the active group were significantly decreased compared with those in the stable group. Furthermore, clinical scores were significantly negatively correlated with the expression levels of SPDEF mRNA and significantly positively correlated with the expression levels of eotaxin-2, which is a biomarker for eosinophilic inflammation on the ocular surface. Cluster analysis classified the patients with $\mathrm{AKC} / \mathrm{VKC}$ into three clusters, and the stable group was divided into two clusters according to the condition of ocular surface mucin. Conclusions. Ocular surface mucin in patients with AKC/VKC is altered in accordance with the clinical severity of the disease.
\end{abstract}

\section{Introduction}

Allergic conjunctival diseases are allergic disorders characterized by eosinophilic inflammation on the ocular surface (consisting of the tear film, conjunctiva, and cornea) and by an immediate hypersensitivity reaction with or without allergen-specific IgE antibodies [1]. Allergic conjunctival diseases consist of allergic conjunctivitis, atopic keratoconjunctivitis (AKC), and vernal keratoconjunctivitis (VKC) [2]; particularly, allergic conjunctival diseases with severe proliferative conjunctival lesions, including giant papillae and gelatinous limbal infiltration, are recognized as refractory and chronic forms of allergic conjunctival diseases that involve severe eosinophilic inflammation and type-2 inflammation via type-2 helper T cell (Th2)-mediated and group 2 innate lymphoid cell-mediated responses [3-5]. Apart from conjunctival proliferative lesions, the severe and chronic forms of atopic and vernal keratoconjunctivitis (AKC/VKC) are also characterized by corneal epithelial damage similar to that clinically observed in dry eye disease [6]. Therefore, maintaining a good tear film is an important aspect of a successful treatment in patients with chronic forms of AKC/VKC.

The three laminar structures of the precorneal tear film are the lipid, aqueous, and mucin layers [7]. Based on clinical tear tests, it has been reported that various allergic inflammation-associated molecules, including eosinophil cationic protein, cytokines, and chemokines, are present in 
tears [3, 8-10], which may cause corneal epithelial damage. Another ocular surface component closely related to corneal epithelial damage is mucin. Mucins are high-molecularweight glycoproteins that contain a central region formed by tandem repeats and $\mathrm{O}$-type carbohydrate chains bound to core proteins [11]. On the mucin-coated ocular surface, MUC5AC (as a gel-forming mucin), MUC1, MUC4, and MUC16 (membrane-associated mucins), as well as MUC7 (derived from the lacrimal gland), are present [12]. However, alterations in the mucin expression pattern on the ocular surface, in the presence of allergic inflammation, are not completely understood.

In patients with bronchial asthma, interleukin-13 (IL13), a Th2 cytokine, has been shown to induce goblet cell hyperplasia in the respiratory epithelium. The induction of goblet cell differentiation is thought to upregulate the SAMpointed domain-containing ETS-like factor (SPDEF) in respiratory epithelial cells $[13,14]$. SPDEF is a transcription factor involved in goblet cell differentiation, and SPDEFknockout mice show a decreased number of goblet cells in intestinal, bronchial, and conjunctival tissues [15]. Therefore, SPDEF in the respiratory epithelium is thought to be induced by intratracheal allergen exposure and Th2 cytokine stimulation $[13,14]$. Furthermore, in allergic conjunctival diseases, goblet cells and MUC5AC secreted from goblet cells are decreased on the ocular surface of patients with $\mathrm{AKC}$ and shield ulcers [16]. In an allergic conjunctival mouse model, MUC5AC mRNA expression in the conjunctiva was found to be reduced after antigen instillation [17]. Therefore, the levels of ocular surface mucin are thought to fluctuate depending on the stage and severity of allergic conjunctival diseases [18].

In this study, we investigated the mRNA expression levels of goblet cell- and mucin-related genes, including SPDEF, MUC5AC, MUC1, MUC4, and MUC16, on the ocular surface in patients with $\mathrm{VKC/AKC}$ by impression cytology (i.e., the clinical ocular surface test). To clarify the allergic inflammatory effect on expression of ocular surface mucin and reduce statistical bias, we compared the results of the ocular surface test between patients with $\mathrm{AKC} / \mathrm{VKC}$ in the active stage and those in the stable stage as controls. We also evaluated whether mRNA expression levels in goblet cell-related and mucin-related genes could provide useful results in ocular allergy tests.

\section{Materials and Methods}

This cross-sectional study was approved by the Institutional Review Board of the Nihon University School of Medicine (approval number: RK-120511-11) and adhered to the tenets of the Declaration of Helsinki. Written informed consent was obtained from all subjects enrolled in this study. This study conforms to the reporting requirements of the STROBE guidelines.

2.1. Patients. In total, 19 patients diagnosed with $\mathrm{AKC}$ or VKC at the Department of Ophthalmology of Nihon University Itabashi Hospital (Tokyo, Japan) were included in this study. The diagnosis of AKC and VKC was based on the Japanese guidelines for allergic conjunctival diseases [2]. The exclusion criteria were as follows: (1) the presence of ocular surface disease, including dry eye disease, other than allergic conjunctival diseases; (2) a history of treatment with systemic antiallergic or anti-inflammatory drugs, such as corticosteroids and immunosuppressants; (3) the use of eye drops to treat dry eyes, such as rebamipide or diquafosol sodium; and (4) the use of contact lenses.

We divided the cohort into 10 patients with stable-stage $\mathrm{AKC/VKC} \mathrm{(stable} \mathrm{group)} \mathrm{and} \mathrm{nine} \mathrm{patients} \mathrm{with} \mathrm{active-stage}$ AKC/VKC (active group), according to their clinical scores. Patients in the stable group had clinical scores of $<100$ points, and patients in the active group had clinical scores of $\geq 100$ points.

2.2. Clinical Scores. The 5-5-5 exacerbation grading scale is a clinical scoring system designed to determine the severity of allergic conjunctival diseases [19]. The clinical score is determined by summing the severe clinical findings, which are given 100 points per item, moderate clinical findings, which are given 10 points per item, and mild clinical findings, which are given 1 point per item. Severe clinical findings include active giant papillae, gelatinous infiltrates in the limbus, exfoliative epithelial keratopathy, shield ulcer, and papillary proliferation at the lower palpebral conjunctiva; moderate findings include blepharitis, papillary proliferation with velvety appearance, Horner-Trantas spots, edema of the bulbar conjunctiva, and superficial punctate keratopathy; and mild findings include papillae at the upper palpebral conjunctiva, follicular lesion at the lower palpebral conjunctiva, hyperemia of the palpebral conjunctiva, hyperemia of the bulbar conjunctiva, and lacrimal effusion. The range of clinical scores on the 5-5-5 exacerbation grading scale is 0 to 555 points.

2.3. Impression Cytology. The targeted eye for impression cytology is the affected eye in unilateral cases or the more severely affected eye in bilateral cases of AKC and VKC. Impression cytology specimens were obtained from the upper tarsal conjunctiva.

Impression cytology using the filter paper method was performed according to our previous reports [20-22]. Since the purpose of this study was to investigate the alterations in ocular surface mucin caused by allergic inflammation, we used the same method of eotaxin-2 mRNA expression level measurement in the upper tarsal conjunctiva as an ocular surface allergy test and thus collected specimens only from the upper tarsal conjunctiva. Briefly, the $5 \mathrm{~mm}$ tip of Schirmer's test paper (Schirmer Tear Production Measuring Strips; Ayumi Pharmaceutical Co., Tokyo, Japan) was used for impression cytology using the filter paper method. Schirmer's test paper was applied to the upper tarsal conjunctiva without local anesthesia, pressed gently using a glass rod, and then removed and preserved in RNA Stabilization Reagent (RNAlater; Qiagen, Hilden, Germany) until total RNA extraction. Then, total RNA in the impression cytology specimen was prepared using the RNeasy ${ }^{\circledR}$ Mini Kit 
(Qiagen) according to the manufacturer's information. cDNA was prepared using the High-Capacity cDNA Reverse-Transcription Kit (Life Technologies Japan, Carlsbad, CA, USA) according to the manufacturer's protocol.

2.4. Real-Time Reverse-Transcription Polymerase Chain Reaction. To detect the expression levels of SPDEF, MUC1, MUC4, MUC5AC, MUC16, and eotaxin-2 mRNA, the realtime reverse-transcription polymerase chain reaction (realtime RT-PCR) method was used. Real-time RT-PCR was performed using the TaqMan gene expression assay (Life Technologies) and predesigned primers/probes, including Hs00159367_m1 (MUC1), Hs00366414_m1 (MUC4), Hs00873651_m1 (MUC5AC), Hs01065189_m1 (MUC16), Hs01026050_m1 (SPDEF), and Hs00171082_m1 (eotaxin-2) (Life Technologies), on a StepOnePlus ${ }^{\mathrm{TM}}$ system (Life Technologies). Target Ct values were normalized to those of GAPDH (Hs99999905_m1) from the same sample. The relative expression levels of each target gene were determined using the $\triangle \triangle \mathrm{CT}$ method.

2.5. Statistical Analysis. All statistical evaluations were performed using the MAC Toukei-Kaiseki v.2 software (Esumi, Tokyo, Japan). Differences in baseline characteristics between the active and stable groups were evaluated using Fisher's exact test or the Mann-Whitney $U$ test. Expression levels of SPDEF, MUC1, MUC4, MUC5AC, and MUC16 mRNA were compared between the stable and active groups using the Mann-Whitney $U$ test. The relationships between the expression levels of SPDEF, MUC16, and eotaxin-2 mRNA were evaluated using a partial correlation coefficient test. Statistical significance was set at $p<0.05$. Cluster analysis based on the Euclidian distance and Ward linkage was used to identify the grouping of chronic allergic conjunctival diseases patients using similar characteristics in terms of clinical scores and expression levels of SPDEF, MUC16, and eotaxin-2 mRNA.

\section{Results}

The demographic and clinical characteristics of the 19 patients in the stable and active groups are shown in Table 1. The average clinical score of the active group was significantly higher than that of the stable group. Eye drops containing an immunosuppressant were administered to all patients, and no patients were treated with steroidcontaining eye drops. None of the patients changed their medications within the 3 months before or after the impression cytology test; even in stable-stage patients, the continuation of medications with immunosuppressive eye drops was necessary to maintain remission.

3.1. Expression Levels of SPDEF, MUC5AC, MUC1, MUC4, and MUC16 mRNA on the OcularSurface. The results of realtime RT-PCR are ratios calculated using the $\Delta \Delta C T$ method, with GAPHD as the endogenous control and the reference patient's measurement as 1 .
The median values of SPDEF mRNA expression levels in the stable and active groups were 12.8 and 1.86 , respectively. $S P D E F$ mRNA expression levels in the active group were significantly lower than those in the stable group $(p<0.05$, Mann-Whitney $U$ test; Figure 1(a)). The median values of $M U C 5 A C$ mRNA expression in the stable and active groups were 3.13 and 0.46 , respectively. MUC5AC mRNA expression levels in the active group were significantly lower than those in the stable group $(p<0.05$, Mann-Whitney $U$ test; Figure 1(b)). SPDEF mRNA expression levels in the stable and active groups were significantly correlated with MUC5AC mRNA expression levels ( $\rho=0.770, p=0.000085$, Spearman's correlation coefficient; Figure 2). Significant differences were not observed between the stable and active groups in the expression of membrane-associated mucin mRNAs, including MUC1, MUC4, and MUC16 (Figure 3).

In addition, eotaxin- 2 mRNA expression levels in the stable and active groups were 30.7 (0.51-69.4) (median (range)) and 106 (3.63-433), respectively. Eotaxin-2 mRNA expression levels in the active group tended to be higher than those in the stable group; and the Mann-Whitney $U$ test showed $p<0.05$ for the one-tailed test and no significant difference in the two-tailed test between stable and active groups.

3.2. Relationship between Clinical Score and Goblet Cell- and Mucin-Related Gene Expression. The correlation between the clinical score and mRNA expression levels of SPDEF, MUC16, and eotaxin-2 was evaluated by determining the partial correlation coefficient. These values are presented in Table 2 . The clinical score was significantly negatively correlated with the expression levels of SPDEF mRNA $(r=-0.484, p=0.049$; Figure $4(\mathrm{a}))$ and significantly positively correlated with the expression levels of eotaxin-2 mRNA ( $r=0.613, p=0.009$; Figure $4(\mathrm{~b})$ ).

With regard to MUC5AC mRNA expression levels, there was no correlation between eotaxin- 2 and MUC5AC mRNA expression levels. In addition, there was a significant negative correlation between clinical score and MUC5AC mRNA expression levels ( $\rho=-0.536$, Spearman's correlation coefficient).

3.3. Cluster Analysis of Patients with ACDs. All patients with active and stable AKC/VKC were evaluated by cluster analysis and grouped into three clusters according to their clinical score and expression levels of SPDEF and MUC16 mRNA. The dendrogram is shown in Figure 5, and the characteristics of the three clusters are listed in Table 3. In the analysis of variance among the three clusters, significant differences were observed in the clinical score and expression levels of SPDEF and MUC5AC mRNA. Cluster 1 included all patients in the active group with low $S P D E F$ and moderate MUC16 mRNA expression. The stable group was divided into two subgroups: one with moderate SPDEF and low MUC16 mRNA expression (Cluster 2) and one with high SPDEF and high MUC16 mRNA expression (Cluster 3). 
TABLE 1: Baseline characteristics of included patients.

\begin{tabular}{lcc}
\hline & Stable group & Active group \\
\hline No. of patients (cases) & 10 & 9 \\
Age (years) (mean \pm SD) & $21.8 \pm 12.3$ & $29.1 \pm 14.9$ \\
Sex (male: female) & $8: 2$ & $9: 0$ \\
Clinical score (points, median) & 12 & 114 \\
Therapeutic use of immunosuppressants (yes/total) & $10 / 10$ & $9 / 9$ \\
\hline
\end{tabular}

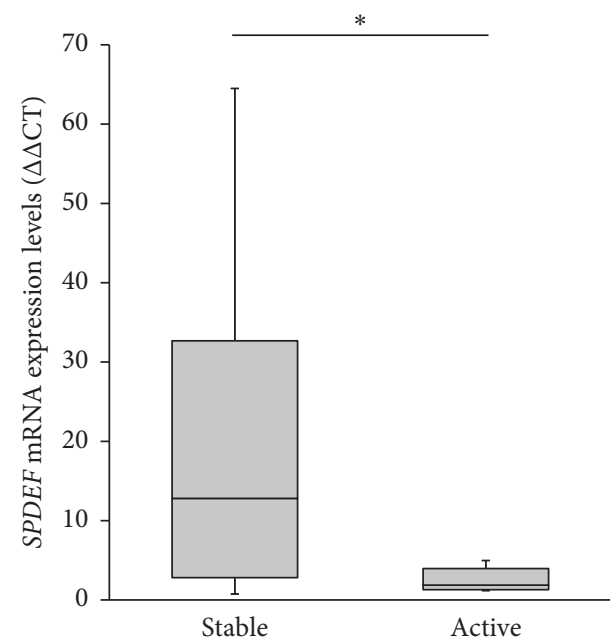

(a)

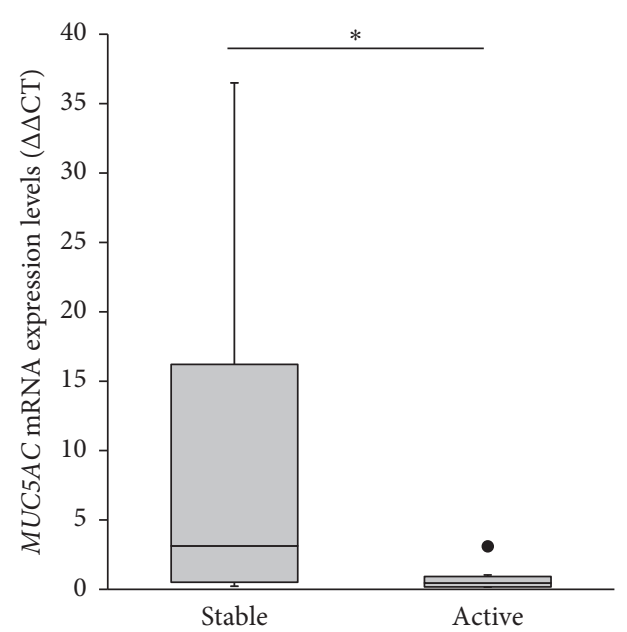

(b)

Figure 1: Messenger RNA expression levels of SPDEF and MUC5AC in stable and active groups. Expression levels of SPDEF (a) and $M U C 5 A C$ (b) mRNA in the active group were significantly higher than those in the stable group. Data are presented as box-whisker plots. Significant differences are indicated as ${ }^{*} p<0.05$.

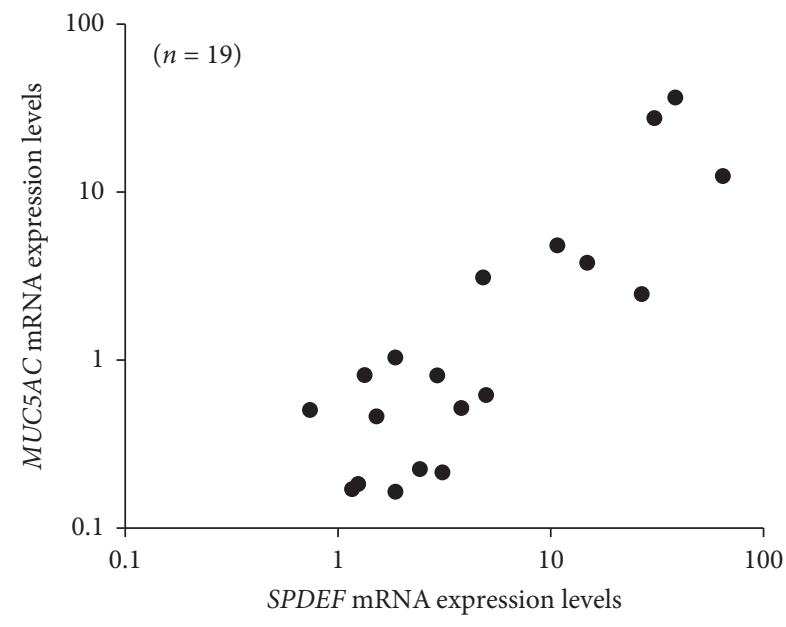

FIGURE 2: Correlation between SPDEF and MUC5AC mRNA expression levels. To examine the correlation, data from the active and stable groups were merged. Spearman's correlation coefficients showed significant correlations between SPDEF and MUC5AC mRNA expression levels ( $\rho=0.770, p=0.000085)$.

\section{Discussion}

We investigated the alterations in ocular surface mucin by performing a clinical ocular surface test using the impression cytology method in patients with $\mathrm{AKC} / \mathrm{VKC}$ in the active stage. The results of the ocular surface test in the active group showed that the expression levels of SPDEF and MUC5AC mRNA were significantly decreased and that the mRNA expression levels of membrane-associated mucins, including MUC1, MUC4, and MUC16, were not significantly altered compared with those in the stable group. Furthermore, clinical scores showed a significant negative correlation with the expression levels of SPDEF mRNA and a positive correlation with the expression levels of eotaxin-2 mRNA. Cluster analysis clarified that patients with stable-stage AKC/VKC could be divided into two subgroups: a subgroup with moderate SPDEF and low MUC16 mRNA expression and a subgroup with high SPDEF and high MUC16 mRNA expression.

SPDEF is a transcriptional factor for goblet cell differentiation. It has been reported that SPDEF expression is increased at sites of goblet cell hyperplasia, which is caused by IL-13 and house-dust-mite allergens, in the respiratory epithelium of adult transgenic mice [12]. Our recent results, which were obtained by performing the clinical ocular surface test, showed that the expression levels of SPDEF mRNA were significantly decreased depending on $\mathrm{AKC} /$ VKC severity and were significantly correlated with the levels of MUC5AC mRNA. Secreted MUC5AC on the ocular surface originates in the conjunctival epithelium and is 


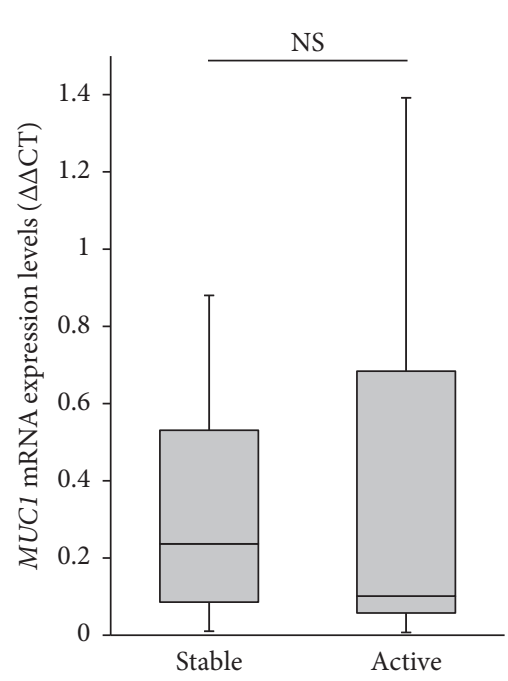

(a)

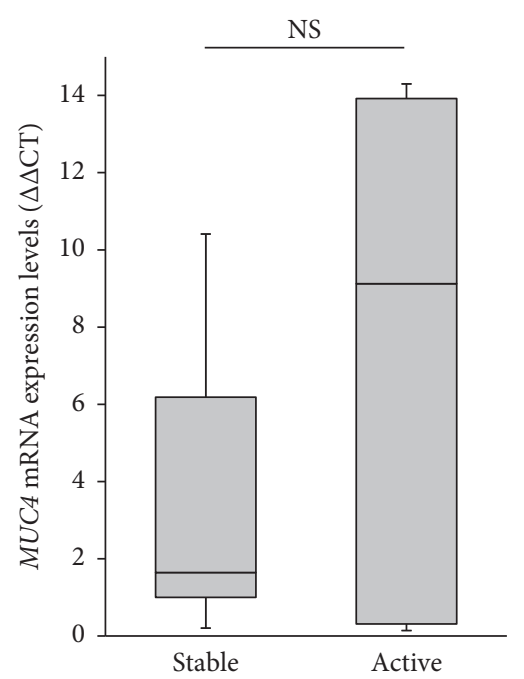

(b)

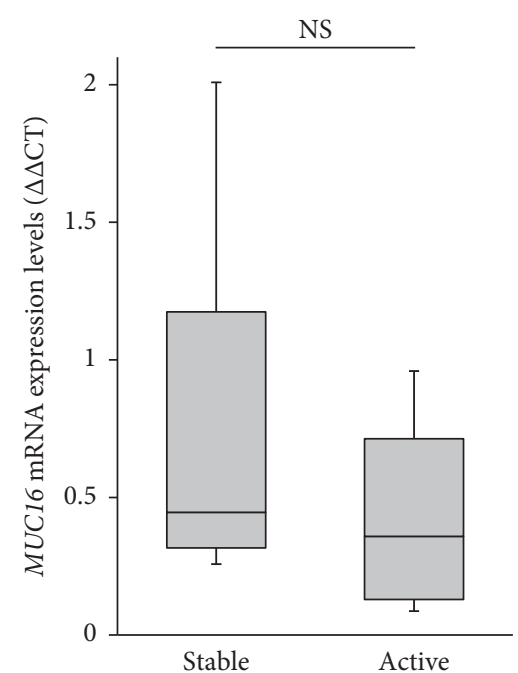

(c)

FIGURE 3: Messenger RNA expression levels of membrane-associated mucin in the stable and active groups. The expression levels of MUC1 (a), MUC4 (b), and MUC16 (c) mRNA showed no significant (NS) differences between the active and stable groups.

TABLE 2: Partial correlation coefficients between gene expression levels and allergic conjunctival disease scores.

\begin{tabular}{lccc}
\hline & Clinical score & SPDEF & MUC16 Eotaxin-2 \\
\hline Clinical score & 1 & & \\
SPDEF & $-0.484^{*}$ & 1 & 1 \\
MUC16 & 0.172 & $0.584^{*}$ & -0.198 \\
Eotaxin-2 & $0.613^{* *}$ & 0.264 & 1 \\
\hline
\end{tabular}

SPDEF, SAM-pointed dominant-containing Ets-like factor. ${ }^{*} p<0.05 ;{ }^{*}{ }^{*} p<0.01$

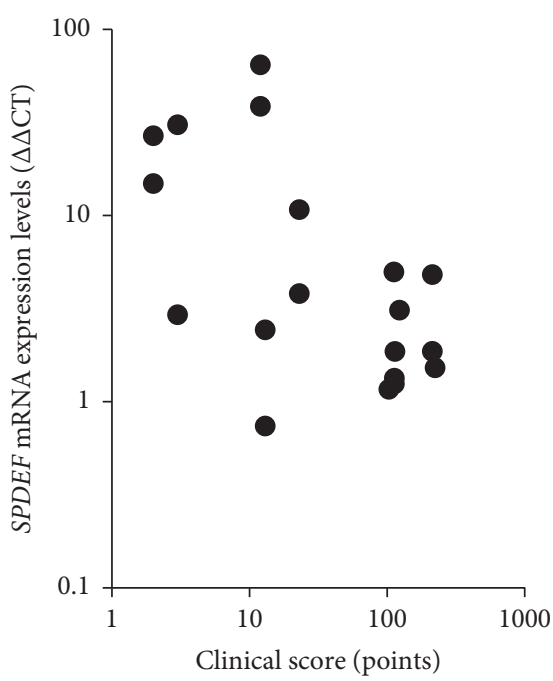

(a)

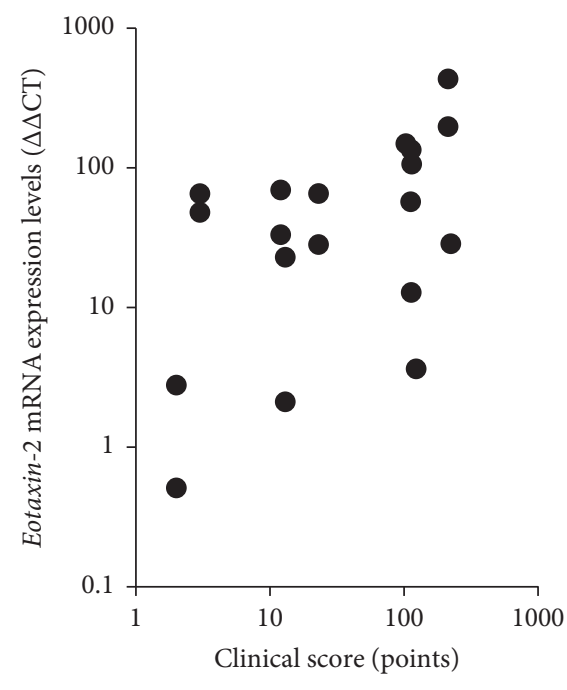

(b)

FIGURE 4: Correlations between clinical score and SPDEF mRNA expression and between clinical score and eotaxin-2 mRNA expression. Partial correlation coefficients showed significant correlations between clinical score and SPDEF mRNA expression levels (a) $(r=-0.484$, $p=0.049)$ and between clinical score and eotaxin-2 mRNA expression levels $(\mathrm{b})(r=0.613, p=0.009)$. 


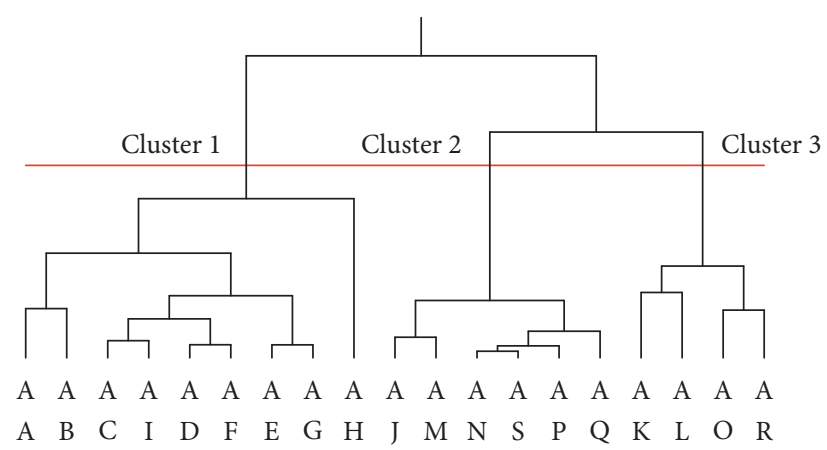

FIGURE 5: Dendrogram of cluster analysis in patients with chronic allergic conjunctival diseases (ACDs). Each of the 19 patients with chronic ACDs were given a code from AA to AS. The active group included those from AA to AI, whereas the stable ACD group included those from AJ to AS. The dendrogram depicts the levels of the hierarchical cluster.

TABLE 3: Characteristics of three cluster groups of allergic conjunctival disease patients.

\begin{tabular}{|c|c|c|c|c|}
\hline & Cluster 1 & Cluster 2 & Cluster 3 & $p$ value* $^{*}$ \\
\hline Clinical score (points) (median (range)) & $114(103-223)$ & $13(2-23)$ & $7.5(3-12)$ & 0.000003 \\
\hline SPDEF (relative expression level) (median (range)) & $1.9(1.24-4.97)$ & $7.3(0.74-14.9)$ & $34.6(2.93-64.5)$ & 0.002 \\
\hline MUC16 (relative expression level) (median (range)) & $0.36(0.09-0.96)$ & $0.33(0.26-0.45)$ & $1.19(1.11-2.00)$ & 0.00008 \\
\hline Eotaxin-2 (relative expression level) (median (range)) & $106(3.63-433)$ & $12.8(0.51-65.5)$ & $56.6(33.2-69.4)$ & 0.134 \\
\hline
\end{tabular}

SPDEF, SAM-pointed dominant-containing Ets-like factor. ${ }^{*}$ Analysis of variance.

derived from goblet cells $[16,23]$. In the conjunctiva of an experimental allergic conjunctivitis mouse model, the number of filled goblet cells reduced, and MUC5AC and MUC4 mRNA levels decreased by the repeated instillation of allergens. Additionally, decreased goblet cell numbers returned to normal levels within $48 \mathrm{~h}$ after the last allergen instillation, and MUC5AC mRNA was found to be restored and increased $24 \mathrm{~h}$ after the last allergen instillation [18]. Regarding the expression of MUC5AC in allergic conjunctival diseases, Dogru et al. [16] reported that MUC5AC, secreted from goblet cells, is decreased in patients with AKC and shield ulcers. Based on our results and those of previous studies, severe allergic inflammation stimulated by allergen exposure on the ocular surface might decrease the number of goblet cells, and a reduction in MUC5AC secretion could elicit dry eye-like clinical findings. Furthermore, alterations in SPDEF and MUC5AC mRNA expression in the conjunctiva are thought to differ from those in the respiratory epithelium. Therefore, the expression levels of SPDEF mRNA, determined by the clinical ocular surface test, might be a useful tool to evaluate goblet cell density in the conjunctiva.

The clinical ocular surface test showed that the expression levels of membrane-associated mucins, including MUC1, MUC4, and MUC16, did not significantly differ between the active and stable groups. In our investigation on ocular surface mucin alterations in Sjögren's syndrome, we reported that SPDEF and MUC16 mRNA expression levels are suitable markers for ocular surface mucin in the patients with Sjögren's syndrome [22]. Therefore, ocular surface mucin in this experiment was assessed with SPDEF and MUC16 mRNA expression levels and dry eye. As a result, cluster analysis revealed that the stable group included a subgroup with moderate SPDEF and low MUC16 mRNA expression levels and a subgroup with high SPDEF and high MUC16 mRNA expression levels. In addition, the active group had low SPDEF and MUC16 mRNA expression levels based on the clinical ocular surface test. These results indicate that chronic allergic conjunctival diseases can be divided into at least three clinical stages depending on the condition of the ocular surface mucin by simultaneously measuring SPDEF and MUC16 through the ocular surface test. In a previous report, Dogru et al. [24] revealed that MUC16 mRNA expression was significantly upregulated, whereas MUC5AC mRNA expression was significantly downregulated in the eyes of patients with AKC compared with that in the eyes of control subjects. This discrepancy in MUC16 mRNA expression might be due to differences in the clinical stages of patients with $\mathrm{AKC} / \mathrm{VKC}$ in each subjective group.

In this study, clinical scores were assessed based on objective findings using the 5-5-5 exacerbation grading scale for allergic conjunctivitis disease [19]. This scoring system is used to evaluate the severity of allergic conjunctival diseases. In the current study, the clinical scores of patients with AKC/VKC were positively correlated with eotaxin-2 mRNA expression [21]. Eotaxin-2 is a chemokine that is strongly expressed in eosinophils [20]. We have previously reported that expression levels of eotaxin-2 mRNA are a useful biomarker to evaluate the severity of eosinophilic inflammation on the ocular surface [21]; we obtained similar results based on the clinical ocular test. In contrast, the clinical scores of patients with AKC/VKC were negatively correlated with the expression levels of SPDEF mRNA. These results indicate that SPDEF is another biomarker of allergic inflammation on the ocular surface and that the goblet cell density in the conjunctiva might be associated with the severity of allergic inflammation on the ocular surface. 
Our study had some limitations. First, the $\Delta \Delta C$ T method used to analyze real-time RT-PCR data is a comparative assay and is not useful for the quantification of the results. The measurement of mRNA expression levels of ocular surface mucin in patients with allergic conjunctival diseases should thus be re-examined by quantitative PCR to determine the normal range of mRNA expression levels. Second, in the current investigation, most of the patients with $\mathrm{AKC}$ and VKC were males; therefore, we were not able to investigate gender differences. Since gender differences in mucin expression on the ocular surface in healthy individuals are controversial, it is necessary to investigate these using a patient group that includes a significant number of female patients with AKC and VKC. Third, the active and stable groups were classified into three clusters based on clinical scores and mRNA expression levels of SPDEF and MUC16. However, in this study, the clinical characteristics of the clusters were not further evaluated. Further studies based on a larger number of patients with allergic conjunctival diseases are necessary to clarify these characteristics. In addition, the usefulness of SPDEF, and MUC16 mRNA as an ocular surface biomarker, should be validated by a population with sufficient sample size, using this study as a pilot study.

\section{Conclusions}

In summary, the levels of ocular surface mucin of patients with chronic ACDs are altered according to the stage and severity of allergic inflammation on the ocular surface. Furthermore, the clinical ocular surface test using impression cytology with filter paper might be useful to quantitatively measure the mRNA expression levels of ocular surface mucin.

\section{Data Availability}

The data that support the findings of this study are available from the corresponding author, S. Y., upon reasonable request.

\section{Conflicts of Interest}

J. S. has previously received honoraria from Santen Pharmaceutical Co., Ltd., Senju Pharmaceutical Co., Ltd., and Alcon Pharmaceuticals, outside the submitted work. S. Y., N. I., Y. T., A. T., and M. H. declare that they have no conflicts of interest.

\section{References}

[1] A. Leonardi, "Allergy and allergic mediators in tears," Experimental Eye Research, vol. 117, pp. 106-117, 2013.

[2] E. Takamura, E. Uchio, N. Ebihara et al., "Japanese guideline for allergic conjunctival diseases," Allergology International, vol. 60, no. 2, pp. 191-203, 2011.

[3] A. Leonardi, S. J. Curnow, H. Zhan, and V. L. Calder, "Multiple cytokines in human tear specimens in seasonal and chronic allergic eye disease and in conjunctival fibroblast cultures," Clinical Experimental Allergy, vol. 36, no. 6, pp. 777-784, 2006.

[4] H. Aso, J. Shoji, Y. Shiraki, N. Inada, and S. Yamagami, "Evaluation of chemokine mRNA expression to assess allergic inflammation of the ocular surface in chronic allergic conjunctival diseases," Cornea, vol. 38, no. 6, pp. 706-712, 2019.

[5] J. Sugita, Y. Asada, W. Ishida et al., "Contributions of interleukin-33 and TSLP in a papain-soaked contact lensinduced mouse conjunctival inflammation model," Immunity, Inflammation and Disease, vol. 5, no. 4, pp. 515-525, 2017.

[6] M. Dogru, N. Nakagawa, K. Tetsumoto, C. Katakami, and M. Yamamoto, "Ocular surface disease in atopic dermatitis," Japanese Journal of Ophthalmology, vol. 43, no. 1, pp. 53-57, 1999.

[7] N. Yokoi and G. A. Georgiev, "Tear film-oriented diagnosis and tear film-oriented therapy for dry eye based on tear film dynamics," Investigative Opthalmology \& Visual Science, vol. 59, no. 14, pp. DES13-DES22, 2018.

[8] A. Secchi, A. Leonardi, and M. Abelson, "The role of eosinophil cationic protein (ECP) and histamine in vernal keratoconjunctivitis," Ocular Immunology and Inflammation, vol. 3, no. 44, pp. 23-28, 1995.

[9] M. Shoji, J. Shoji, and N. Inada, "Clinical severity and tear biomarkers, eosinophil cationic protein and CCL23, in chronic allergic conjunctival diseases," Seminars in Ophthalmology, vol. 33, no. 3, pp. 325-330, 2018.

[10] J. Shoji, H. Aso, and N. Inada, "Clinical usefulness of simultaneous measurement of the tear levels of CCL17, CCL24, and IL-16 for the biomarkers of allergic conjunctival disorders," Current Eye Research, vol. 42, no. 5, pp. 677-684, 2017.

[11] R. R. Hodges and D. A. Dartt, "Tear film mucins: front line defenders of the ocular surface; comparison with airway and gastrointestinal tract mucins," Experimental Eye Research, vol. 117, pp. 62-78, 2013.

[12] I. K. Gipson, "Distribution of mucins at the ocular surface," Experimental Eye Research, vol. 78, no. 3, pp. 379-388, 2004.

[13] K.-S. Park, T. R. Korfhagen, M. D. Bruno et al., "SPDEF regulates goblet cell hyperplasia in the airway epithelium," Journal of Clinical Investigation, vol. 117, no. 4, pp. 978-988, 2007.

[14] G. Chen, T. R. Korfhagen, Y. Xu et al., "SPDEF is required for mouse pulmonary goblet cell differentiation and regulates a network of genes associated with mucus production," The Journal of Clinical Investigation, vol. 119, no. 10, pp. 29142924, 2009.

[15] C. K. Marko, B. B. Menon, G. Chen, J. A. Whitsett, H. Clevers, and I. K. Gipson, "Spdef null mice lack conjunctival goblet cells and provide a model of dry eye," The American Journal of Pathology, vol. 183, no. 1, pp. 35-48, 2013.

[16] M. Dogru, N. Asano-Kato, M. Tanaka et al., "Ocular surface and MUC5AC alterations in atopic patients with corneal shield ulcers," Current Eye Research, vol. 30, no. 10, pp. 897-908, 2005.

[17] K. S. Kunert, A. M. Keane-Myers, S. Spurr-Michaud, A. S. Tisdale, and I. K. Gipson, "Alteration in goblet cell numbers and mucin gene expression in a mouse model of allergic conjunctivitis," Investigative Ophthalmology \& Visual Science, vol. 42, no. 11, pp. 2483-2489, 2001.

[18] D. A. Dartt and S. Masli, "Conjunctival epithelial and goblet cell function in chronic inflammation and ocular allergic inflammation," Current Opinion in Allergy \& Clinical Immunology, vol. 14, no. 5, pp. 464-470, 2014.

[19] J. Shoji, N. Inada, and M. Sawa, "Evaluation of novel scoring system named 5-5-5 exacerbation grading scale for allergic 
conjunctivitis disease," Allergology International, vol. 58, no. 4, pp. 591-597, 2009.

[20] N. Inada, J. Shoji, Y. Shiraki, H. Aso, and S. Yamagami, "Histamine $\mathrm{H} 1$ and $\mathrm{H} 4$ receptor expression on the ocular surface of patients with chronic allergic conjunctival diseases," Allergology International, vol. 66, no. 4, pp. 586-593, 2017.

[21] Y. Shiraki, J. Shoji J, and N. Inada, "Clinical usefulness of monitoring expression levels of CCL24 (eotaxin-2) mRNA on ocular surface in patients with vernal keratoconjunctivitis and atopic keratoconjunctivitis," Journal of Ophthalmology, vol. 2016, Article ID 3573142, 5 pages, 2016.

[22] J. Shoji, N. Inada, A. Tomioka, and S. Yamagami, "Assessment of mucin-related gene alterations following treatment with rebamipide ophthalmic suspension in Sjögren's syndromeassociated dry eyes," PLoS One, vol. 15, no. 11, p. e0242617, 2020.

[23] Y. Hori, "Secreted mucins on the ocular surface," Investigative Opthalmology \& Visual Science, vol. 59, no. 14, pp. DES151DES156, 2018.

[24] M. Dogru, Y. Matsumoto, N. Okada et al., "Alterations of the ocular surface epithelial MUC16 and goblet cell MUC5AC in patients with atopic keratoconjunctivitis," Allergy, vol. 63, no. 10, pp. 1324-1334, 2008. 